\title{
CARACTERÍSTICAS DE LAS INFECCIONES DEL TORRENTE SANGUÍNEO EN PACIENTES ADULTOS DE DOS CENTROS DE TERCER NIVEL DE CÓRDOBA, ARGENTINA.
}

\section{CHARACTERISTICS OF BLOODSTREAM INFECTIONS IN ADULT PATIENTS OF TWO THIRD LEVEL CENTERS OF CÓRDOBA, ARGENTINA.}

\begin{abstract}
Emanuel José Saad ${ }^{1,2,6}$, Diego Federico Baenas ${ }^{1}$, Cecilia Soledad Boisseau ${ }^{3}$, Mercedes de Jesús García1, Silvana Alejandra Nuñez ${ }^{1}$, Domingo Cesar Balderramo ${ }^{2,4}$, Daniela Hernández y Juan Pablo Caeiro ${ }^{2,3}$.
\end{abstract}

\section{Resumen:}

Introducción: Las infecciones del torrente sanguíneo (ITS) están asociadas a elevada morbimortalidad. Objetivo: Determinar características epidemiológicas, microbiológicas y clínicas de ITS adquiridas en la comunidad (ITS-AC) e intrahospitalarias (ITS-IH). Métodos: Se analizaron retrospectivamente eventos de bacteriemia y fungemia en dos hospitales de tercer nivel entre abril de 2009 y agosto de 2013. Resultados: Se identificaron 1150 eventos de 53\% correspondieron a ITS-AC. El 61\% de microorganismos aislados fueron gram negativos. Los patógenos más frecuentes fueron Escherichia coli en ITS-AC y Klebsiella pneumoniae en ITS-IH. Staphylococcus aureus fue el gram positivo más frecuente. Las principales comorbilidades fueron enfermedad renal (39\%) y neoplasias (38\%). El 26,8\% de ITS fueron primarias. Los focos infecciosos más frecuentes fueron respiratorio (17\%) y urinario $(16,9 \%)$. Un elevado porcentaje de gram negativos en ITS-AC e ITS-IH fueron resistentes a ampicilina sulbactam (40,2\% y 57,5\%), cefalotina $(36,7 \%$ y $46,8 \%)$, trimetoprima-sulfametoxazol (32,8\% vs $35,5 \%)$ y ciprofloxacina $(24,6 \%$ y $35,3 \%)$. Staphylococcus aureus meticilino resistente fue más frecuente en ITS-IH (31,4\% vs $11,8 \%, p=0,007)$. Conclusiones: Las características clínicas y epidemiológicas de ITS fueron similares a las reportadas por otros estudios latinoamericanos. Pero observamos algunas diferencias en los perfiles de susceptibilidad antimicrobiana. Resaltamos la importancia de la vigilancia epidemiológica local para elegir tratamientos empíricos apropiados.

Palabras clave: adultos; bacteriemia; epidemiología; antiinfecciosos.

\section{Abstract:}

Background: Bloodstream infections $(\mathrm{BI})$ are associated with high morbidity and mortality. Objetive: To determine epidemiological, microbiological and clinical features of community (CA-BI) and nosocomial bloodstream infections (N-BI). Methods: Bacteremia and fungemia events were retrospectively analyzed in two third-level hospitals between April 2009 and August 2013. Results: We identified 1150 events of bloodstream infections, $53.2 \%$ were CA-BI. Gram negative microorganisms were isolated in $61 \%$. The most frequent pathogens were Escherichia coli in CA-BI and Klebsiella pneumoniae in N-BI. Staphylococcus aureus was the most frequent gram positive organism. The main comorbidities were renal disease (39\%) and malignancy $(38 \%)$. There were $26.8 \%$ of primary bloodstream infections, and the main infection foci included respiratory (17.04\%) and urinary tract (16.86\%). A high percentage of gram-negative bacteria of $\mathrm{CA}-\mathrm{BI}$ and $\mathrm{N}-\mathrm{BI}$ were resistance to ampicillin sulbactam (40.2\% and $57.5 \%)$, cephalothin (36.7\% and $46.8 \%)$, trimethoprim-sulfamethoxazole $32.8 \%$ vs $35.5 \%)$ and ciprofloxacin (24.6\% and $35.3 \%)$. Methicillin-resistant Staphylococcus aureus were more frequently into ITS-IH (31.4\% vs $11.8 \%, p=0.007)$. Conclusions: Clinical and epidemiological characteristics of $\mathrm{CA}-\mathrm{BI}$ and $\mathrm{N}-\mathrm{BI}$ were similar to those reported by other Latin-American studies. We observed some differences in antimicrobial resistance profiles. We emphasize the importance of local epidemiological surveillance to choose appropriate empirical treatments.

Keywords: adults; bacteremia; epidemiology; anti-infective agents.

\footnotetext{
1. Médico. Departamento de Medicina Interna, Hospital Privado Universitario de Córdoba. Córdoba, Argentina.

2. Instituto Universitario de Ciencias Biomédicas de Córdoba. Córdoba, Argentina.

3. Médico. Departamento de Enfermedades Infecciosas, Hospital Privado Universitario de Córdoba. Córdoba, Argentina

4.Médico. Departamento de Gastroenterología, Hospital Privado Universitario de Córdoba. Córdoba, Argentina

5. Bioquímica. Departamento de Microbiología, Hospital Privado Universitario de Córdoba. Córdoba, Argentina

6.Email de contacto: emanuelsaad@hotmail.com
} 
Las infecciones del torrente sanguíneo (ITS) constituyen una importante causa de morbimortalidad a nivel mundial. La incidencia de bacteriemia y fungemia varía entre 3 a 28 episodios por cada 1000 ingresos hospitalarios. ${ }^{1,2,3}$ La mortalidad asociada oscila entre 14 y $38 \%$ y depende de múltiples factores: agente etiológico, foco infeccioso asociado, comorbilidades del paciente, lugar de adquisición y tratamiento antimicrobiano empírico adecuado, entre otros ${ }^{4,5,6}$. Se asocian a un incremento en los días de hospitalización y a un gran impacto económico en los sistemas sanitarios ${ }^{7}$.

En los últimos años se han observado modificaciones en la epidemiología de los microorganismos responsables de las ITS. En la década del 1980 existía un franco predominio de las bacterias gram negativas en sepsis intrahospitalaria y posteriormente aumentó significativamente el número de episodios provocados por gram positivos y hongos. Esto no ocurrió en las infecciones adquiridas en la comunidad en las que los gram negativos continúan siendo los principales causantes de bacteriemia ${ }^{1,4,8}$. Asimismo, se ha descripto un considerable aumento en la resistencia antimicrobiana ${ }^{6,9,10}$.

Los estudios de vigilancia epidemiológica locales constituyen una herramienta de gran utilidad para fomentar el uso prudente y adecuado de los antimicrobianos, y controlar la aparición y diseminación de los mecanismos de resistencia ${ }^{3}$.

El objetivo del presente estudio fue determinar las características epidemiológicas, microbiológicas y clínicas de los episodios de bacteriemia y fungemia, adquiridas en la comunidad e intrahospitalarias.

\section{Métodos}

Se realizó un estudio retrospectivo en dos hospitales universitarios de tercer nivel de la ciudad de Córdoba, Argentina (Hospital Privado Universitario de Córdoba y Hospital Raúl Ángel Ferreyra). Estas instituciones poseen un promedio de 12890 y 2516 admisiones por año, respectivamente. Ambos centros comparten el sistema de historia clínica electrónica y procesan las muestras en el mismo Departamento de Microbiología. Se identificaron todos los hemocultivos (HC) informados como positivos durante un período de 4 años (abril de 2009 hasta agosto de 2013), utilizando la base de datos del servicio de Microbiología. Se revisaron las historias clínicas de todos los eventos de bacteriemia y fungemia verdaderas en pacientes mayores a 18 años de edad. Se excluyeron los eventos de hemocultivos considerados como contaminación. Se compararon las ITS verdaderas adquiridas en la comunidad con las intrahospitalarias, registrando las características demográficas, comorbilidades de los pacientes, foco infeccioso asociado, y los aislamientos microbiológicos con sus respectivos perfiles de resistencia antimicrobiana. Se analizó la mortalidad asociada a las ITS en cada grupo.

\section{Definiciones:}

-Hemocultivo positivo verdadero: crecimiento de uno o más microorganismos en al menos una muestra de HC, exceptuando a aquellos agentes microbiológicos que son potenciales contaminantes de la piel (Propionibacterium spp, Bacillus spp, Staphylococcus coagulasa negativa, Corynebacterium spp, Streptococcus grupo viridans), en cuyo caso debían cumplir al menos uno de los siguientes criterios: a) desarrollo en dos o más muestras de $\mathrm{HC}$; b) aislamiento del mismo microorganismo en otro sitio considerado como fuente de la infección; c) infección intravascular en presencia de temperatura $>38^{\circ} \mathrm{C} 0<36^{\circ} \mathrm{C}$ sin otro patógeno aislado, que motivó el inicio de terapia antimicrobiana específica para el mismo.

-Mismo episodio de ITS: aquellos HC con idénticos aislamientos microbiológicos y sensibilidad antimicrobiana, tomados del mismo paciente y en el mismo momento, y referidos al mismo foco infeccioso. -Hemocultivos polimicrobianos: aislamiento de dos o más especies de microorganismos, en uno o más $\mathrm{HC}$ tomados en un mismo paciente y momento, acordes a la definición de $\mathrm{HC}$ positivos verdaderos.

-ITS adquirida en la comunidad (ITS-AC): cuando el HC fue obtenido dentro de las primeras 48 horas de hospitalización, sin mediar ninguna actividad asistencial previa ${ }^{11}$.

-ITS intrahospitalaria (ITS-IH): cuando el hemocultivo fue adquirido luego de 48 horas de hospitalización en un paciente internado por una razón distinta a la ITS, o si esta ocurrió en el período hasta las 72 horas después del alta². 
-ITS primaria o sin foco: aislamiento de microorganismos en los $\mathrm{HC}$, cumpliendo con la definición de HC positivo verdadero, sin identificación de un foco infeccioso probable.

-Infección respiratoria baja: Incluye: a) traqueobronquitis, radiografía de tórax sin infiltrados alveolares y presencia de 2 de las siguientes: 1)fiebre $>38^{\circ} \mathrm{C}$, 2)tos, 3)expectoración purulenta y/o aislamiento de microorganismo en secreciones respiratorias, 4)sibilancias o roncus; b) neumonía, radiografía con infiltrado alveolar y uno de los siguientes: 1) rales crepitantes o matidez, 2) expectoración purulenta y/o aislamiento de microorganismo en secreciones respiratorias, 3) $\mathrm{HC}$ positivo.

-Infección urinaria: a) urocultivo positivo, con al menos $10^{5} \mathrm{UFC}$ con fiebre $>38^{\circ} \mathrm{C}$ y síntomas urinarios (disuria, urgencia, polaquiuria, dolor abdominal); b) presencia de síntomas urinarios, urocultivo con $>10^{2}$ UFC y leucocituria>10/campo; c) bacteriemia luego de la manipulación del sistema urinario (uretrostomía, cistostomía, colocación de catéter pig-tail o nefrostomía en árbol urinario, biopsia prostática transrrectal); d) prostatitis.

-Infección asociada a catéter: a) cultivo de punta de catéter con >15 UFC Técnica de Maki asociado a hemocultivo con aislamiento del mismo microorganismo y uno de los siguientes: 1) fiebre $>38^{\circ} \mathrm{C}$, 2) dolor regional, 3) eritema, 4) calor; b) hemocultivo del catéter positivo 2 horas antes del HC, con mismo aislamiento de microorganismo ${ }^{12}$.

-Infección de sitio quirúrgico: infección que ocurre dentro de los 30 días posteriores a una cirugía (en caso de tratarse de procedimiento quirúrgico profundo, se extiende hasta 90 días), asociado a drenaje purulento de dicho sitio o aislamiento del mismo microorganismo en $\mathrm{HC}$ y líquido de drenaje de sitio quirúrgico ${ }^{13}$.

-Endocarditis: criterios de Duke modificados ${ }^{14}$.

-Infección de piel o partes blandas: a) drenaje purulento, vesículas o ampollas, b) presencia de 2 elementos de la tétrada de Celsius (dolor, eritema, aumento de la temperatura, edema) y cultivo de secreción y/o HC positivo.

-Osteomielitis: Una de las siguientes: 1) cultivo de hueso positivo; 2) anatomía patológica que confirma osteomielitis; 3) HC positivo y diagnóstico de osteomielitis por imágenes, en ausencia de otro foco infeccioso probable ${ }^{15}$.

-Meningitis: a) líquido cefalorraquídeo con tinción gram o cultivo positivo y/o detección de antígeno positivo de microorganismos; o b) líquido cefalorraquídeo patológico en presencia de síntomas meníngeos (cefaleas, rigidez de nuca, parálisis de pares craneales, irritabilidad).

-Peritonitis: dolor abdominal, acompañado o no con defensa y reacción peritoneal, asociados a la presencia de líquido peritoneal purulento o un recuento de polimorfonucleares mayor a 250/mm3.

-Colecistitis/colangitis: fiebre o evidencia de respuesta inflamatoria en el laboratorio, asociada a elevación de enzimas hepáticas o alteración en la vía biliar (dilatación, cambios estructurales, litiasis coledociana, stent biliar, etc.) y/o de la vesícula biliar (engrosamiento anormal de paredes y/o características inflamatorias en anatomía patológica) ${ }^{16}$.

-Foco gastrointestinal: gastroenteritis (diarrea, con o sin vómitos, en presencia de fiebre $>38^{\circ} \mathrm{C}$ ), enterocolitis.

\section{Métodos microbiológicos.}

Se utilizó el sistema de monitoreo continuo de HC, utilizando la tecnología de sensor-fluorescente (BACTEC 9240, Becton Dickinson). La identificación y test de susceptibilidad antimicrobiana fue realizada con el método Vitek 2/bioMérieux.

\section{Análisis estadístico}

Las variables continuas fueron expresadas como media \pm desvío estándar y rango, y las categóricas como números y porcentajes. Se calcularon los intervalos de confianza del $95 \%$. Se compararon las características de los eventos de ITS-AC con ITS-IH, utilizando el test t de 2 muestras independientes para las variables continuas y la prueba de chi $^{2}$ o test exacto de Fisher, según correspondiese, en las variables categóricas. Se consideró como significativo un valor de probabilidad de 2 colas menor a 0.05 . El análisis estadístico fue desarrollado con el paquete estadístico SPSS 17.0 (SPSS, Inc., Chicago, IL). 


\section{Resultados}

Durante el período de estudio se realizaron un total de $37858 \mathrm{HC}$, de los cuales 2681 (7\%) fueron positivos. Se excluyeron 468 HC (316 en menores de 18 años, 115 considerados contaminación, y 37 correspondientes a 22 eventos de bacteriemia y fungemia por falta de datos en la historia clínica).

Se incluyeron $2132 \mathrm{HC}$ que correspondieron a 1150 eventos de ITS, representando 6 eventos de ITS cada 1000 admisiones por año entre las dos instituciones. Se identificaron 25 eventos de ITS polimicrobianas (2,17\% del total de eventos de ITS).

EI 53,2\% de los eventos fueron ITS-AC. Hubo más pacientes de sexo masculino tanto en ITS-AC como en ITS-IH, con mayor predominio aún en el grupo ITS-IH (64,1\% vs $57,2 \%, p=0,016)$. El promedio de edad de los pacientes fue mayor en los eventos de ITS-AC $(6, .2 \pm 16,8$ vs $58,2 \pm 16,6, p=0,002)$. La mayoría de los pacientes con ITS se encontraban en el rango etario entre 50 y 70 años $(44,1 \%$ ITS-AC vs $44,6 \%$ ITS-IH). Hubo mayor frecuencia de pacientes pertenecientes al grupo etario de 30-49 años en los eventos de ITS-IH ( $21,9 \%$ vs $15,8 \%, p=0,008)$ y del grupo etario de mayores de 69 años en ITS-AC ( $34 \%$ vs $27 \%, p=0,009)$.

Tabla 1: Características demográficas de los pacientes con infecciones del torrente sanguíneo.

\begin{tabular}{|c|c|c|c|}
\hline & $\begin{array}{l}\text { Adquirido en la } \\
\text { Comunidad }\end{array}$ & Intrahospitalario & $\mathbf{p}$ \\
\hline $\mathrm{N}^{\circ}$ de HC Positivos, $\mathrm{n}(\%)$ & $1155(54.2)$ & $977(45.8)$ & \\
\hline $\mathrm{N}^{\circ}$ de Eventos de $\mathrm{HC}$ positivos, $\mathrm{n}(\%)$ & $612(53.2)$ & $538(46.8)$ & \\
\hline \multicolumn{4}{|l|}{ Hospital } \\
\hline Hospital Ferreyra, $\mathrm{n}(\%)$ & $96(15.7)$ & $68(12.6)$ & 0.14 \\
\hline Hospital Privado, $\mathrm{n}(\%)$ & $516(84.3)$ & $470(87.4)$ & 0.14 \\
\hline Sexo masculino, $\mathrm{n}(\%)$ & $350(57.2)$ & $345(64.1)$ & 0.016 \\
\hline Edad (años), media \pm DE & $61.22 \pm 16.80$ & $58.15 \pm 16.61$ & 0.002 \\
\hline \multicolumn{4}{|l|}{ Grupos Etarios, n(\%) } \\
\hline 18-29 años & $37(6)$ & $35(6.5)$ & 0.74 \\
\hline 30-49 años & $97(15.8)$ & $118(21.9)$ & 0.008 \\
\hline $50-69$ años & $270(44.1)$ & $240(44.6)$ & 0.866 \\
\hline$>69$ años & $208(34)$ & $145(27)$ & 0.009 \\
\hline Mortalidad, $\mathrm{n}(\%)$ & $98(16)$ & $177(32.9)$ & $<0.001$ \\
\hline
\end{tabular}

$\boldsymbol{H C}$ : Hemocultivos. $\boldsymbol{D E}$ : Desvío Estandar

En relación a las comorbilidades, la enfermedad renal fue la más frecuente con 450 eventos (39,1\%), de los cuales un $18 \%$ se encontraban en terapia de reemplazo renal. Hubo 440 eventos (38,3\%) en pacientes con neoplasias, con predominio significativo en el grupo de ITS-IH (43,5\% vs 33,7\%; p<0,001). La asociación con insuficiencia cardíaca, cardiopatía isquémica y diabetes mellitus tipo I también fue mayor con el grupo de ITS-IH de manera significativa. El 15,3\% de los eventos se produjeron en pacientes trasplantados de órganos sólidos (principalmente trasplantados renales), siendo más frecuentes en ITS-IH (17,5\% vs 13,4\%; $\mathrm{p}=0,055)$. Hubo 68 eventos en pacientes trasplantados de médula ósea, y en este subgrupo también se mantuvo la predominancia en las ITS-IH $(8,9 \%$ vs $3,3 \% ; p<0,001)$. Los pacientes con neutropenia febril representaron el $13 \%$ del total de eventos, mayoritariamente en ITS-IH $(p<0,001)$. Las comorbilidades más frecuentes en el grupo de ITS-AC fueron el síndrome de inmunodeficiencia adquirida, trasplante de páncreas y tabaquismo. 
Tabla 2: Comorbilidades de pacientes con infecciones del torrente sanguíneo.

\begin{tabular}{|c|c|c|c|}
\hline Comorbildiades & $\begin{array}{c}\text { Adquirido en la } \\
\text { Comunidad N/ (\%) }\end{array}$ & $\begin{array}{c}\text { Intrahospitalario } \mathrm{N} / \\
(\%)\end{array}$ & $\mathbf{p}$ \\
\hline Enfermedad arterial periférica & $62(10.1)$ & $57(10.6)$ & 0.796 \\
\hline ICC & $87(14.2)$ & 104(19.3) & 0.02 \\
\hline Cardiopatía isquémica & $67(10.9)$ & 103(19.1) & $<0.001$ \\
\hline Enfermedad Renal & $229(37.4)$ & $221(41.07)$ & 0.204 \\
\hline Daño renal agudo & $51(8.3)$ & $50(9.3)$ & 0.56 \\
\hline ERC & $178(29.1)$ & $171(31.8)$ & 0.3205 \\
\hline Terapia de reemplazo renal & $102(16.7)$ & $106(19.7)$ & 0.182 \\
\hline$H D$ & $90(15)$ & $100(19.7)$ & 0.077 \\
\hline$D P$ & $12(2)$ & $6(1.1)$ & 0.249 \\
\hline Diabetes Mellitus & $134(21.9)$ & $120(22.3)$ & 0.867 \\
\hline DBT II & $124(20.3)$ & $100(18.6)$ & 0.474 \\
\hline$D B T I$ & $10(1.6)$ & 20(3.7) & 0.027 \\
\hline Cáncer & $206(33.7)$ & $234(43.5)$ & $<0.001$ \\
\hline Mama & $20(3.3)$ & $17(3.2)$ & 0.917 \\
\hline Cérvix & $8(1.3)$ & $6(1.1)$ & 0.7671 \\
\hline Próstata & $9(1,5)$ & $11(2)$ & 0.45 \\
\hline Hematológico & $75(12.3)$ & $119(22.1)$ & $<0.001$ \\
\hline Gastrointestinal & $19(3.1)$ & $35(6.5)$ & 0.007 \\
\hline Hepato-biliar & $12(2)$ & $11(2)$ & 0.919 \\
\hline Páncreas & $18(2.9)$ & $5(0.9)$ & 0.015 \\
\hline Renal-Vía urinaria & $14(2.3)$ & $6(1.1)$ & 0.1292 \\
\hline Otros & $31(5.1)$ & $24(4.5)$ & 0.6318 \\
\hline \multicolumn{4}{|l|}{ Tabaquismo } \\
\hline Ex Tabaquista & $173(28.3)$ & $173(32.2)$ & 0.151 \\
\hline Tabaquista & $91(14.9)$ & $60(11.2)$ & 0.064 \\
\hline EPOC & $41(6.7)$ & $26(4.8)$ & 0.177 \\
\hline Asma & $27(4.4)$ & $25(4.6)$ & 0.8482 \\
\hline Enfermedad Neurológica & $69(11.3)$ & $73(13.6)$ & 0.238 \\
\hline Tx de órgano sólido & $82(13.4)$ & $94(17.5)$ & 0.055 \\
\hline Cardíaco & $6(1)$ & $7(1.3)$ & 0.60 \\
\hline Hepático & $6(1)$ & $14(2.6)$ & 0.0358 \\
\hline Pulmonar & $0(0)$ & $3(0.6)$ & 0.20 \\
\hline Renal & $72(11.8)$ & $70(13)$ & 0.52 \\
\hline Páncreas & $4(0.7)$ & $16(3)$ & 0.0027 \\
\hline TMO & $20(3.3)$ & $48(8.9)$ & $<0.001$ \\
\hline Quimioterapia & $115(18.8)$ & $128(23.8)$ & 0.038 \\
\hline Consumo de Corticoides o inmunosupresores & $130(21.2)$ & $156(29)$ & 0.002 \\
\hline SIDA & $10(1.6)$ & $3(0.6)$ & 0.099 \\
\hline Enfermedad Hepática & $41(6.7)$ & $31(5.8)$ & 0.51 \\
\hline Neutropenia febril & $50(8.2)$ & $102(19)$ & $<0.001$ \\
\hline
\end{tabular}

Hubo 308 eventos (26,8\%) de ITS sin foco, con predominio en ITS-IH (34,9\% vs $19,6 \% ; p<0,001)$. Los focos infecciosos identificados más frecuentes fueron el respiratorio (17\%) y urinario (16,9\%). Las infecciones relacionadas a la herida quirúrgica, las asociadas a catéter, las de origen intraabdominal y las bacteriemias intradiálisis se observaron más en el grupo ITS-IH. Por otro lado, las infecciones de tejidos blandos, 
INFECCIONES DEL TORRENTE SANGUÍNEO EN ADULTOS.

urinarias, colangitis, colecistitis y peritonitis predominaron en ITS-AC. Fallecieron 215 pacientes (18,7\%), con mayor mortalidad en ITS-IH $(32,9 \%$ vs $16 \%, p<0,001)$.

Tabla 3: Principales enfermedades infecciosas en los pacientes con infecciones del torrente sanguíneo.

\begin{tabular}{lccc}
\hline \multicolumn{1}{c}{ Enfermedades infecciosas } & $\begin{array}{c}\text { Adquirido en la Comunidad N/ } \\
(\mathbf{\%})\end{array}$ & Intrahospitalario N/ (\%) & p \\
\hline Bacteriemia primaria & $120(19.6)$ & $188(34,9)$ & $<0.001$ \\
Infección de tejidos blandos & $55(9)$ & $16(3)$ & $<0.001$ \\
Infección respiratoria & $106(17.3)$ & $90(16.7)$ & 0.79 \\
Endocarditis. & $18(2.9)$ & $7(1.3)$ & 0.057 \\
Herida quirúrgica & $7(1.1)$ & $31(5.8)$ & $<0.001$ \\
ITU & $140(22.9)$ & $54(10)$ & $<0.001$ \\
Infecciones asociadas a catéter & $24(3.9)$ & $47(8.7)$ & $<0.001$ \\
Colecistitis/Colangitis & $59(9.6)$ & $13(2.4)$ & $<0.001$ \\
Intraabdominal & $34(5.6)$ & $49(9.1)$ & 0.020 \\
Peritonitis & $22(3.6)$ & $5(0.9)$ & 0.003 \\
Bacteriemia asociada a hemodiálisis & $0(0)$ & $28(5.2)$ & $<0.001$ \\
Otros focos infecciosos & $27(4.4)$ & $10(1.9)$ & 0.014 \\
\hline
\end{tabular}

Se aislaron microorganismos gram negativos en 701 eventos (61\%), siendo responsables del 67,5\% de las ITS-IH ( $p<0,001)$. Los patógenos más frecuentes fueron Escherichia coli, con mayor presentación en ITSAC $(p<0,001)$. Los bacilos gram negativos más frecuentes en ITS-IH fueron Klebsiella pneumoniae, Serratia marcescens y Acinetobacter baumannii $(\mathrm{p}<0,001)$. La mayoría de los aislamientos de microorganismos gram positivos provenían de la comunidad $(43,6 \%$ vs $32,9 \% ; p<0,001)$. Los más frecuentes fueron Staphylococcus aureus, Staphylococcus coagulasa negativa, Streptococcus pneumoniae y Enterococcus faecalis. Las bacterias gram positivas que predominaron en el grupo de ITS-IH con respecto a ITS-AC fueron Enterococcus faecium ( $9,4 \%$ vs $0,7 \% ; p<0,001)$ y Staphylococcus epidermidis $(17,4 \%$ vs $8,2 \%, p<0,005)$. Por el contrario, los microorganismos del género Streptococcus, principalmente S. pneumoniae, se hallaron más en el grupo ITS-AC.

Se identificaron 33 episodios de fungemia, representando el 2,87\% del total de eventos de ITS, y fueron más frecuentes en ITS-IH (4,8\% vs 1,1\%; $p<0,001)$. La mayoría de los aislamientos fúngicos correspondían a hongos levaduriformes, principalmente del género Candida spp. En los pacientes con ITS intrahospitalaria el $15,4 \%$ de los aislamientos correspondió a Candida albicans, mientras que todas las candidemias de los episodios adquiridos en la comunidad fueron provocados por Candida no albicans.

Tabla 4: Principales aislamientos microbiológicos de los pacientes con infecciones del torrente sanguíneo.

\begin{tabular}{lccc}
\hline \multicolumn{1}{c}{ Microorganismos } & $\begin{array}{c}\text { Adquirido en la } \\
\text { Comunidad N/ (\%) }\end{array}$ & $\begin{array}{c}\text { Intrahospitalario N/ } \\
(\mathbf{\%})\end{array}$ & $\mathbf{p}$ \\
\hline Gram Negativos & $\mathbf{3 3 8}(\mathbf{5 5 . 2})$ & $\mathbf{3 6 3}(\mathbf{6 7 . 5})$ & $<\mathbf{0 . 0 0 1}$ \\
Acinetobacter & $6(1.8)$ & $26(7.2)$ & $\mathbf{0 . 0 0 1}$ \\
$\quad$ Acinetobacter baumannii & $3(0.9)$ & $22(6.1)$ & $<\mathbf{0 . 0 0 1}$ \\
Otros $\quad 3(0.9)$ & $4(1.1)$ & 1 \\
Enterobacter & $14(4.1)$ & $13(3.6)$ & 0.7 \\
$\quad$ Enterobacter cloacae & $7(2.1)$ & $10(2.8)$ & 0.556 \\
$\quad$ Enterobacter aerogenes & $6(1.8)$ & $2(0.6)$ & 0.164 \\
$\quad$ Otros & $1(0.3)$ & $1(0.3)$ & 1 \\
Escherichia coli & $179(53)$ & $89(24.5)$ & $<\mathbf{0 . 0 0 1}$ \\
Klebsiella & $55(16.3)$ & $105(28.9)$ & $<\mathbf{0 . 0 0 1}$ \\
Klebsiella oxytoca & $7(2.1)$ & $7(1.9)$ & 0.808 \\
$\quad$ Klebsiella pneumoniae & $48(14.2)$ & $98(27)$ & $<\mathbf{0 . 0 0 1}$
\end{tabular}




\begin{tabular}{|c|c|c|c|}
\hline Pseudomonas & $33(9.8)$ & $45(12.4)$ & 0.268 \\
\hline Pseudomonas aeruginosa & $32(9.5)$ & 42(11.6) & 0.365 \\
\hline Otras Pseudomonas & $1(0.3)$ & $3(0.8)$ & 0.625 \\
\hline Burkholderia cepacia & $9(2.7)$ & $15(4.1)$ & 0.285 \\
\hline Proteus & $6(1.8)$ & $9(2.5)$ & 0.520 \\
\hline Proteus mirabilis & $6(1.8)$ & $7(1.9)$ & 0.881 \\
\hline Proteus vulgaris & $0(0)$ & $2(0.6)$ & 0.5 \\
\hline Serratia marcescens & $9(2.7)$ & $33(9.1)$ & $<0.001$ \\
\hline Stenotrophomonas maltophilia & $9(2.7)$ & $12(3.3)$ & 0.618 \\
\hline Salmonella spp & $9(2.7)$ & $4(1.1)$ & 0.126 \\
\hline Otros gram negativos & $9(2.7)$ & $28(7.7)$ & 0.0028 \\
\hline Gram Positivos & 267(43.6) & 149(32.9) & $<0.001$ \\
\hline Enterococcus & $30(11.2)$ & $28(18.8)$ & $\mathbf{0 . 0 3 3}$ \\
\hline Enterococcus faecalis & $27(10.1)$ & $12(8.1)$ & 0.49 \\
\hline Enterococcus faecium & $2(0.7)$ & $14(9.4)$ & $<0.001$ \\
\hline Otros & $1(0.4)$ & $2(1.3)$ & 0.292 \\
\hline Staphylococcus coagulasa positiva & $77(28.8)$ & $51(34.2)$ & 0.254 \\
\hline Staphylococcus aureus & $76(28.5)$ & $51(34.2)$ & 0.221 \\
\hline Staphylococcus schleiferi & $1(0.4)$ & $0(0)$ & 1 \\
\hline Staphylococcus coagulasa negativa & $46(17.2)$ & $41(27.5)$ & 0.013 \\
\hline Staphylococcus epidermidis & $22(8.2)$ & $26(17.4)$ & 0.005 \\
\hline Staphylococcus haemolyticus & $3(1.1)$ & $4(2.7)$ & 0.255 \\
\hline Staphylococcus hominis & $9(3.4)$ & $2(1.3)$ & 0.341 \\
\hline Staphylococcus warneri & $5(1.9)$ & $1(0.7)$ & 0.427 \\
\hline Otros & $7(2.6)$ & $8(5.4)$ & 0.150 \\
\hline Streptococcus & 111(41.6) & $27(18.1)$ & $<0.001$ \\
\hline Streptococcus agalactiae & $10(3.7)$ & $3(2)$ & 0.394 \\
\hline Streptococcus dysgalactiae & $8(3)$ & $0(0)$ & 0.055 \\
\hline Streptococcus pneumoniae & $58(21.7)$ & $8(5.4)$ & $<0.001$ \\
\hline Streptococcus mitis & $8(3)$ & $9(6)$ & 0.133 \\
\hline Otros & $27(10.1)$ & $7(4.7)$ & $\mathbf{0 . 0 5 3}$ \\
\hline Otros gram positivos & $3(1.1)$ & $2(1.3)$ & 1 \\
\hline HONGOS & $7(1.1)$ & $26(4.8)$ & $<0.001$ \\
\hline Hongos levaduriformes & $7(100)$ & $25(96.2)$ & 1 \\
\hline Candida & $6(85.7)$ & $22(84.6)$ & 1 \\
\hline Candida albicans & $0(0)$ & $4(15.4)$ & 0.555 \\
\hline Candida glabrata & $1(14.3)$ & $4(15.4)$ & 1 \\
\hline Candida krusei & $0(0)$ & $4(15.4)$ & 0.55 \\
\hline Candida parapsilosis & $5(71.4)$ & $4(15.4)$ & 0.009 \\
\hline Candida spp & $0(0)$ & $6(23.1)$ & 0.301 \\
\hline Otros hongos levaduriformes & $1(14.3)$ & $3(11.5)$ & 1 \\
\hline Hongos filamentosos & $0(0)$ & $1(3.8)$ & 1 \\
\hline
\end{tabular}

Se analizó comparativamente la susceptibilidad a antimicrobianos en los grupos de ITS-AC e ITS-IH ocasionados por bacterias gram negativas, y se observó un elevado porcentaje de resistencia a ampicilina sulbactam $(40,2 \%$ y $57,3 \%$ respectivamente), cefalotina $(36,7 \%$ y $46,8 \%)$, trimetoprima sulfametoxazol $(32,8 \%$ vs $35,5 \%)$ y ciprofloxacina $(24,6 \%$ y $35,3 \%)$. Se identificaron 116 gérmenes productores de betalactamasa de espectro extendido (BLEE), representando el 16,54\% del total de los bacilos gram negativos aislados. Los eventos provocados por bacterias productoras de BLEE fueron más frecuentes en ITS-IH (22\% vs 10,7\%, p<0,001). 
Tabla 5: Resistencia antimicrobiana de los microorganismos gram negativos aislados en pacientes con infecciones del torrente sanguíneo.

\begin{tabular}{|c|c|c|c|}
\hline $\begin{array}{l}\text { Microorganismos y } \\
\text { antibióticos testeados }\end{array}$ & $\begin{array}{c}\text { Adquirido en la } \\
\text { Comunidad N/ (\%) }\end{array}$ & $\begin{array}{c}\text { Intrahospitalario N/ } \\
(\%)\end{array}$ & $\mathbf{p}$ \\
\hline \multicolumn{4}{|l|}{ Gram Negativos } \\
\hline AMS & $136(40.2 \%)$ & $208(57.3 \%)$ & $<0.001$ \\
\hline Cefalotina & $124(36.7 \%)$ & $170(46.8 \%)$ & 0.007 \\
\hline Ceftazidima & $35(10.4 \%)$ & $52(14.3 \%)$ & 0.11 \\
\hline Cefotaxima & $19(5.6 \%)$ & $12(3.3 \%)$ & 0.136 \\
\hline Cefepime & $44(13 \%)$ & $111(30.6 \%)$ & $<0.001$ \\
\hline Ciprofloxacina & $83(24.6 \%)$ & $128(35.3 \%)$ & 0.002 \\
\hline Amikacina & $8(2.4 \%)$ & $26(7.2 \%)$ & 0.003 \\
\hline Gentamicina & $56(16.6 \%)$ & $74(20.4 \%)$ & 0.194 \\
\hline Imipenem & $10(3 \%)$ & $36(9.9 \%)$ & $<0.001$ \\
\hline Meropenem & $11(3.3 \%)$ & $40(11 \%)$ & $<0.001$ \\
\hline PTZ & $27(8 \%)$ & $101(27.8 \%)$ & $<0.001$ \\
\hline TMP/SMX & $111(32.8 \%)$ & $129(35.5 \%)$ & 0.452 \\
\hline Colistina & $4(1.2 \%)$ & $2(0.6 \%)$ & 0.436 \\
\hline BLEE & $36(10.7)$ & $80(22.0)$ & $<0.001$ \\
\hline \multicolumn{4}{|l|}{ Acinetobacter baumannii } \\
\hline Ceftazidima & $3(100 \%)$ & $16(100 \%)$ & 0.554 \\
\hline Cefepime & $3(100 \%)$ & $21(95.5 \%)$ & 1 \\
\hline Ciprofloxacina & $3(100 \%)$ & $21(95.5 \%)$ & 1 \\
\hline Amikacina & $1(33.3 \%)$ & $5(22.7 \%)$ & 1 \\
\hline Imipenem & $3(100 \%)$ & $22(100 \%)$ & 1 \\
\hline Meropenem & $3(100 \%)$ & $22(100 \%)$ & 1 \\
\hline PTZ & $3(100 \%)$ & $21(100 \%)$ & 1 \\
\hline Colistina & $1(33.3)$ & $0(0)$ & 0.12 \\
\hline \multicolumn{4}{|l|}{ Enterobacter cloacae } \\
\hline AMS & $7(100)$ & $10(100)$ & 1 \\
\hline Cefalotina & $7(100)$ & $10(100)$ & 1 \\
\hline Ceftazidima & $1(14.3)$ & $1(10)$ & 1 \\
\hline Cefotaxima & $1(14.3)$ & $0(0)$ & 0.412 \\
\hline Cefepime & $1(14.3)$ & $2(20 \%)$ & 1 \\
\hline Ciprofloxacina & $0(0)$ & $2(20)$ & 0.485 \\
\hline Amikacina & $0(0)$ & $0(0)$ & 1 \\
\hline Gentamicina & $1(14.3)$ & $2(20)$ & 1 \\
\hline Imipenem & $0(0)$ & $0(0)$ & 1 \\
\hline Meropenem & $0(0)$ & $0(0)$ & 1 \\
\hline PTZ & $0(0)$ & $2(20 \%)$ & 0.485 \\
\hline TMP/SMX & $1(14.3)$ & $2(20)$ & 1 \\
\hline \multicolumn{4}{|l|}{ Escherichia coli } \\
\hline AMS & $80(44.7)$ & $58(65.2)$ & 0.002 \\
\hline Cefalotina & $76(42.5)$ & $51(57.3)$ & 0.022 \\
\hline Ceftazidima & $16(8.9)$ & $8(9)$ & 0.989 \\
\hline Cefotaxima & $11(6.1)$ & $4(4.5)$ & 0.58 \\
\hline Cefepime & $21(11.7)$ & $18(20.2)$ & 0.06 \\
\hline Ciprofloxacina & $54(30.2)$ & $44(49.4)$ & 0.002 \\
\hline Amikacina & $3(1.7)$ & $3(3.4)$ & 0.402 \\
\hline Gentamicina & $34(19)$ & $13(14.6)$ & 0.374 \\
\hline
\end{tabular}




\section{Imipenem}

Meropenem

PTZ

TMP/SMX

Klebsiella pneumoniae

AMS

Cefalotina

Ceftazidima

Cefotaxima

Cefepime

Ciprofloxacina

Amikacina

Gentamicina

Imipenem

Meropenem

PTZ

TMP/SMX

Pseudomonas

Ceftazidima

Cefepime

Ciprofloxacina

Amikacina

Gentamicina

Imipenem

Meropenem

PTZ

Colistina

Burkholderia cepacia

Ceftazidima

Minociclina

Meropenem

TMP/SMX

Serratia marcescens

$\begin{array}{lc}\text { Ceftazidima } & 0(0) \\ \text { Cefepime } & 0(0) \\ \text { Ciprofloxacina } & 0(0) \\ \text { Amikacina } & 0(0) \\ \text { Gentamicina } & 0(0) \\ \text { Imipenem } & 0(0) \\ \text { Meropenem } & 0(0) \\ \text { PTZ } & 0(0) \\ \text { TMP/SMX } & 0(0) \\ \text { Colistina } & 2(22.2)\end{array}$

$0(0)$

$0(0)$

$12(13.5)$

60(67.4)

23(47.9)

19(39.6)

$5(10.4)$

$5(10.4)$

12(25)

15(31.3)

$1(2.1)$

13(27.1)

$1(2.1)$

1(2.1)

13(27.1)

17(35.4)

2(6.3)

3(9.4)

$3(9.4)$

1(3.1)

3(9.4)

4(12.5)

5(15.6)

$0(0)$

$0(0)$

$0(0)$

$0(0)$

1(11.1)

1(11.1)

66(67.3)

59(60.2)

11(11.2)

5(5.1)

41(41.8)

36(36.7)

6(6.1)

31(31.6)

1(1)

1(1)

44(44.9)

41(41.8)

7(16.7)

10(23.8)

11(26.2)

3(7.1)

8(19)

10(23.8)

9(21.4)

12(28.6)

0(0)

1(6.7)

1(6.7)

8(53.3)

7(46.7)

3(9.1)

12(36.4)

6(18.2)

7(21.2)

11(33.3)

3(9.1)

$0(0)$

6(18.2)

3(9.1)

1(3)

\section{1}

1

0.027

0.001

0.024

0.019

0.883

0.298

0.047

0.514

0.426

0.574

0.55

0.55

0.038

0.456

0.284

0.106

0.067

0.629

0.331

0.218

0.528

0.001

1

\section{1}

1

0.08

0.178

1

0.41

0.312

0.314

0.083

1

1

0.312

1

0.11

AMS: Ampicilina-Sulbactam. TMP/SMX: Trimetoprima-sulfametoxazol. PTZ: Piperacilina tazobactam. BLEE: Betalactamasa de espectro extendido.

Los Staphylococcus aureus meticilino resistentes fueron más frecuentemente responsables de ITS-IH $(31,4 \%$ vs $11,8 \%, p=0,007)$, mientras que los Staphylococcus epidermidis fueron muy frecuentes tanto en el grupo de las ITS-AC como en las ITS-IH, pero sin diferencias significativas $(63,6 \%$ vs $76,9 \%$ respectivamente, $p=0,313$ ). 
Tabla 6: Resistencia antimicrobiana de los microorganismos gram positivos aislados en las infecciones del torrente sanguíneo.

\begin{tabular}{|c|c|c|c|}
\hline $\begin{array}{c}\text { Microorganismos y antibióticos } \\
\text { testeados }\end{array}$ & $\begin{array}{c}\text { Adquirido en la } \\
\text { Comunidad N/ (\%) }\end{array}$ & Intrahospitalario N/ (\%) & $\mathbf{p}$ \\
\hline \multicolumn{4}{|l|}{ Staphylococcus aureus } \\
\hline Oxacilina & $9(11.8 \%)$ & $16(31.4 \%)$ & 0.007 \\
\hline Ciprofloxacina & $5(6.6 \%)$ & $8(15.7 \%)$ & 0.097 \\
\hline Gentamicina & $10(13.2 \%)$ & $13(25.5 \%)$ & 0.077 \\
\hline Clindamicina & $28(36.8 \%)$ & $18(35.3 \%)$ & 0.86 \\
\hline TMP/SMX & $2(2.6 \%)$ & $2(3.9 \%)$ & 1 \\
\hline Vancomicina & $0(0)$ & $0(0)$ & 1 \\
\hline Minociclina & $0(0)$ & $0(0)$ & 1 \\
\hline \multicolumn{4}{|l|}{ Staphylococcus epidermidis } \\
\hline Oxacilina & $14(63.6 \%)$ & $20(76.9 \%)$ & 0.313 \\
\hline Ciprofloxacina & $9(40.9 \%)$ & $18(69.2 \%)$ & 0.049 \\
\hline Gentamicina & $12(54.5 \%)$ & $12(46.2 \%)$ & 0.56 \\
\hline Clindamicina & $13(59.1 \%)$ & $19(73.1 \%)$ & 0.306 \\
\hline TMP/SMX & $9(40.9 \%)$ & $12(46.2 \%)$ & 0.715 \\
\hline Vancomicina & $0(0)$ & $1(3.8 \%)$ & 1 \\
\hline Minociclina & $1(405 \%)$ & $1(3.8 \%)$ & 1 \\
\hline \multicolumn{4}{|l|}{ Enterococcus faecalis } \\
\hline Ampicilina & $0(0)$ & $1(8.3)$ & 0.308 \\
\hline Ciprofloxacina & $0(0)$ & $1(8.3)$ & 0.308 \\
\hline Gentamicina & $10(37)$ & $2(16.7)$ & 0.276 \\
\hline Vancomicina & $0(0)$ & $0(0)$ & 1 \\
\hline
\end{tabular}

\section{Discusión}

La ITS constituye un cuadro clínico grave que se asocia a una elevada morbimortalidad. ${ }^{1-5,7,17}$ En nuestro estudio la frecuencia de la mortalidad fue de $16 \%$ en los eventos adquiridos en la comunidad y $33 \%$ en el medio hospitalario. Estos datos son similares a los de otros reportes ${ }^{1-5,7}$. A pesar de los avances tecnológicos, el incremento en la resistencia de los microorganismos es significativamente mayor al descubrimiento de nuevos antibióticos ${ }^{18}$. Por este motivo resulta indispensable el conocimiento de la epidemiología del ámbito de trabajo ${ }^{1-5,7}$. especialmente debido a que existen estudios de bacteriemias en cuidados intensivos que demostraron que la tasa de mortalidad aumenta del $30 \%$ al $60 \%$ cuando el tratamiento inicial es inapropiado ${ }^{19}$.

En el presente estudio, la mayoría de las ITS fueron adquiridas en la comunidad al igual que en otros estudios clínicos recientes ${ }^{20}$. Numerosos trabajos han reportado que en 16 a $22 \%$ de los casos no es posible determinar el foco infeccioso de origen ${ }^{4,3,11}$, situación que observamos en 19,6\% de las ITS-AC, y $35 \%$ en los eventos de ITS-IH. En concordancia con publicaciones nacionales e internacionales, cuando se pudo identificar el foco primario, las infecciones respiratorias y urinarias fueron las más frecuentes ${ }^{4,3,8,11}$.

Desde el punto de vista clínico y sanitario es fundamental conocer la frecuencia de infecciones asociadas a catéteres intravasculares. Los episodios de bacteriemias asociadas a catéter rondan entre 4-27\% de las bacteriemias nosocomiales, con una mortalidad que oscila entre el $8-15 \%{ }^{21}$.

En nuestra muestra, los eventos de ITS fueron más frecuentes en pacientes mayores de 50 años de edad y en aquellos que presentaban comorbilidades que generan mayor inmunocompromiso. Observamos $39 \%$ de eventos asociados a enfermedad renal, y aproximadamente la mitad se encontraban en terapia de reemplazo renal. No encontramos diferencias significativas al analizar esta comorbilidad entre los grupos de ITS-AC e ITS-IH. También hallamos un elevado porcentaje de pacientes con antecedente de neoplasias (muchos de ellos bajo tratamiento quimioterápico), tratamiento inmunosupresor, diabetes mellitus y trasplantados de órgano sólido y médula ósea. Estos factores se relacionan con mayor número de internaciones, generando una mayor exposición a diversos patógenos y aumentando la predisposición a desarrollar ITS posteriores ${ }^{11}$. Además, estos pacientes tienen mayor riesgo de eventos de neutropenia febril, 
con el consiguiente aumento de la mortalidad ${ }^{1-7,12}$. Todos los factores de riesgo mencionados han sido reportados previamente $\mathrm{s}^{3,11,22,23}$.

En nuestro estudio los microorganismos gram negativos fueron los agentes etiológicos más frecuentes en ambos grupos. En ITS-AC, E. coli fue responsable del 53\% de los aislamientos microbiológicos y $K$. pneumoniae fue el principal agente en ITS-IH. Al igual que en otras publicaciones regionales, $E$. coli fue frecuentemente resistente a ampicilina sulbactam, cefalosporinas de primera generación, trimetoprimasulfametoxazol y ciprofloxacina. Así mismo, Klebsiella pneumoniae también fue frecuentemente resistente a piperacilina-tazobactam, ciprofloxacina y cefepime ${ }^{17,24}$. El número de eventos nosocomiales provocados por $E$. coli fue significativamente menor al de la comunidad, y se observó que $P$. aeruginosa, $S$. marcescens y $A$. baumannii fueron responsables del $27.9 \%$ de los eventos en este grupo. Estas bacterias adquieren gran interés porque se asocian a una mortalidad mayor a $25 \%$ debido su patogenicidad y al incremento en la resistencia antimicrobiana ${ }^{24,25}$.

Existe una creciente preocupación por la emergencia de bacterias gram negativas multirresistentes, como es el caso de Escherichia coli, Klebsiella pneumoniae y Proteus mirabilis, que representan cerca de 15\%, $26 \%$ y $32 \%$ respectivamente ${ }^{5,24,26}$. En algunos estudios se ha observado que los microorganismos productores de BLEE representan 6 al $11 \%$ de los bacilos gram negativos responsables de ITS, cifras similares a las observadas en nuestro análisis ${ }^{11,26}$.

Por otro lado, observamos un mayor número de eventos provocados por gérmenes gram positivos en ITSIH respecto a las ITS-AC. En diferentes reportes se ha observado este fenómeno, y en algunos países desarrollados los gram positivos constituyen actualmente la primera causa de ITS. 5,27,28 Estos datos se repiten en estudios regionales de ITS-AC en los que Streptococcus pneumoniae y Staphylococcus aureus representan los principales microorganismos aislados ${ }^{11,23}$. En un estudio multicéntrico realizado en EEUU, se observó que del total de bacteriemias por Staphylococcus aureus en adultos, el 43,9\% eran meticilino resistente de los cuales $77,1 \%$ eran adquiridos en la comunidad ${ }^{1}$. En estudios nacionales, como en nuestro caso, la frecuencia de Staphylococcus aureus meticilino resistentes adquiridos en la comunidad representan $14 \%$ aproximadamente ${ }^{11}$.

La frecuencia de ITS polimicrobianas fue menor a la reportada en la mayoría de los estudios, donde se encuentra de 7\%. Este grupo de pacientes particularmente está asociado a una mayor mortalidad ${ }^{1,2,11}$. Los episodios de fungemia son responsables de $1 \%$ de las ITS-AC y asciende al 7\% en ITS-IH nuestro medio encontramos menor frecuencia a nivel nosocomial.

Este trabajo tiene las limitaciones propias de un diseño retrospectivo. Además, debemos destacar que sólo reportamos la prevalencia de resistencia in vitro a los antibióticos más utilizados, y no se evaluó la tasa de fracaso terapéutico.

En conclusión, este análisis constituye una aproximación microbiológica necesaria para el conocimiento de las infecciones del torrente sanguíneo. Consideramos necesarios estudios y registros permanentes de los eventos de ITS a mayor escala en nuestro país, para poder extrapolar los datos a la realidad clínica diaria y así optimizar el tratamiento antimicrobiano.

\section{Bibliografía}

1. Franco Moreno Al, Casallo Blanco S, Marcos Sánchez F, Sánchez Casado M, Gil Ruiz MT, Martínez de la Casa Muñoz AM. Estudio de las bacteriemias en el Servicio de Medicina Interna de un hospital de grupo 2: Análisis de los tres últimos años. An Med Interna. 2005;22(5):217-21.

2. Gambra M, Flores J, Ramírez K, Palma S, Zitko P, Valenzuela MT et al. Incidencia y mortalidad de bacteriemia en un hospital clínico docente en Santiago de Chile. Rev Med Chil. 2012;140(7):859-66.

3. Nazar JR, Lavados A, Daher O, Bischoff MC. Análisis microbiológico, epidemiológico y evolución clínica de los pacientes con bacteriemia en el Hospital Zonal de Esquel en el período 2007-2009. Rev Argent Microbiol. 2010;42(3):151-64.

4. Beltrán MA, Rodríguez E, Sorvik D, Gil R, Guerrera J, Bertolini P et al. Estudio clínico y epidemiológico de pacientes adultos con hemocultivos positivos. Medicina (B Aires). 2002;62(1):13-9.

5. Luzzaro F, Ortisi G, Larosa M, Drago M, Brigante G, Gesu G. Prevalence and epidemiology of microbial pathogens causing bloodstream infections: results of the OASIS multicenter study. Diagn Microbiol Infect Dis. 2011;69(4):3639.

6. Wisplinghoff H, Bischoff T, Tallent SM, Seifert H, Wenzel RP, Edmond MB. Nosocomial bloodstream infections in US hospitals: analysis of 24,179 cases from a prospective nationwide surveillance study. Clin Infect Dis. 2004;39(3):309-17. 
7. Karchmer AW. Nosocomial bloodstream infections: organisms, risk factors, and implications. Clin Infect Dis. 2000;31(4):S139-43.

8. Hernández-Roca JJ, García-Vázquez E, Hernández A, Canteras M, Herrero JA, Cascales E et al. Bacteriemias en un hospital de segundo nivel: Estudio epidemiológico, análisis de factores pronósticos asociados a mortalidad y estimación de su coste económico. Rev Esp Quimioter. 2013;26(2):119-27.

9. Muñoz P, Cruz AF, Rodríguez-Créixems M, Bouza E. Gram-negative bloodstream infections. Int J Antimicrob Agents. 2008;32(1):S10-4.

10. Pop-Vicas A, Tacconelli E, Gravenstein S, Lu B, D'Agata EM. Influx of multidrug-resistant, gram-negative bacteria in the hospital setting and the role of elderly patients with bacterial bloodstream infection. Infect Control Hosp Epidemiol. 2009;30(4):325-31.

11. Artico MJ, Rocchi M, Gasparotto A, Ocaña Carrizo V, Navarro M, Mollo V et al. Bacteriemias de origen comunitario en pacientes adultos que acuden al servicio de urgencias de un hospital universitario. Rev Argent Microbiol. 2012;44(1):10-5.

12. Mermel LA, Allon M, Bouza E, Craven DE, Flynn P, O'Grady NP et al. Clinical practice guidelines for the diagnosis and management of intravascular catheter-related infection: 2009 Update by the Infectious Diseases Society of America. Clin Infect Dis. 2009;49(1):1-45.

13. Horan TC, Andrus M, Dudeck MA. CDC/NHSN surveillance definition of health care-associated infection and criteria for specific types of infections in the acute care setting. Am J Infect Control. 2008;36(5):309-32.

14.Durack DT, Lukes AS, Bright DK. New criteria for diagnosis of infective endocarditis: utilization of specific echocardiographic findings. Duke Endocarditis Service. Am J Med. 1994;96(3):200-9.

15. Centers for Disease Control and Prevention (CDC). Enero 2017. CDC/NHSN surveillance definitions for specific types of infections. [Online] CDC website. Disponible en https://www.cdc.gov/nhsn/pdfs/pscmanual/17pscnosinfdef_current.pdf. Consultado el 26 de agosto de 2017.

16. Kiriyama S, Takada T, Strasberg SM, Solomkin JS, Mayumi T, Pitt HA et al. New diagnostic criteria and severity assessment of acute cholangitis in revised Tokyo Guidelines. J Hepatobiliary Pancreat Sci. 2012;19(5):548-56

17. Koupetori M, Retsas T, Antonakos N, Vlachogiannis G, Perdios I, Nathanail C et al. Bloodstream infections and sepsis in Greece: over-time change of epidemiology and impact of de-escalation on final outcome. BMC Infect Dis. 2014;14:272.

18. Marston HD, Dixon DM, Knisely JM, Palmore TN, Fauci AS. Antimicrobial Resistance. JAMA. 2016;316(11):1193204.

19. Ibrahim EH, Sherman G, Ward S, Fraser VJ, Kollef MH. The influence of inadequate antimicrobial treatment of bloodstream infections on patient outcomes in the ICU setting. Chest. 2000;118(1):146-55.

20. Laupland KB, Church DL. Population-Based Epidemiology and Microbiology of Community-Onset Bloodstream Infections. Clin Microbiol Rev. 2014;27(4):647-64.

21. León C, Sánchez MA, Lucena F. Infección por catéter: antes y después de la Conferencia de Consenso. Enferm Infecc Microbiol Clin. 1997;15(3):27-32.

22. Rabagliati BR, Fuentes LG, Orellana UE, Oporto CJ, Domínguez MI, Benítez GR et al. Etiology of febrile neutropenia episodes among cancer patients from Hospital Clinico Universidad Catolica, Santiago-Chile. Rev Chilena Infectol. 2009;26(2):106-13.

23. Sanz Carabaña P, Ramos Martínez A, Asensio Vegas A, García Navarro MJ, Linares Rufo M. Mortalidad y factores pronósticos en pacientes hospitalizados por bacteriemia adquirida en la comunidad. An Med Interna. 2006; 23(2): 66-72.

24.Tsay RW, Siu LK, Fung CP, Chang FY. Characteristics of bacteremia between community-acquired and nosocomial Klebsiella pneumoniae infection: risk factor for mortality and the impact of capsular serotypes as a herald for community-acquired infection. Arch Intern Med. 2002;162(9):1021-7.

25.Pollack, M. Pseudomonas aeruginosa. En: Mandel, G., Bennett, J.E., Dolin, R. Editor. Principles and Practice of Infectious Diseases, 5th edition. Churchill Livingstone, Philadelphia 2000; 2: 2310-2335.

26. Peña C, Pujol M, Ardanuy C, Ricart A, Pallarés R, Liñares J et al. An outbreak of hospital-acquired Klebsiella pneumoniae bacteraemia, including strains producing extended-spectrum beta-lactamase. J Hosp Infect. 2001;47(1):53-9.

27.Diekema DJ, Beekmann SE, Chapin KC, Morel KA, Munson E, Doern GV. Epidemiology and outcome of nosocomial and community-onset bloodstream infection. J Clin Microbiol. 2003;41(8):3655-60.

28. Sader HS, Jones RN, Andrade-Baiocchi S, Biedenbach DJ; SENTRY Participants Group (Latin America). Fouryear evaluation of frequency of occurrence and antimicrobial susceptibility patterns of bacteria from bloodstream infections in Latin American medical centers. Diagn Microbiol Infect Dis. 2002;44(3):273-80. 